\title{
AN OSCILLATION CRITERION FOR SECOND ORDER NONLINEAR DIFFERENTIAL EQUATIONS
}

\author{
JAMES S. W. WONG
}

\begin{abstract}
An oscillation crierion is given for the second order nonlinear differential equation $x^{\prime \prime}+a(t)|x|^{\gamma} \operatorname{sgn} x=0, \gamma>0$, where the coefficient $a(t)$ is not assumed to be nonnegative for all large values of $t$. The result involves a condition obtained by Kamenev for the linear differential equation.
\end{abstract}

Consider the second order nonlinear differential equation

$$
x^{\prime \prime}+a(t)|x|^{\gamma} \operatorname{sgn} x=0, \quad \gamma>0,
$$

where $a(t) \in C[0, \infty)$. We restrict our attention to solutions of (1) which exist on some ray $\left[t_{0}, \infty\right)$, where $t_{0} \geq 0$ may depend on the particular solution. Such a solution is said to be oscillatory if it has arbitrarily large zeros. Equation (1) is called oscillatory if all such solutions are oscillatory. For a general discussion on nonlinear oscillation problems, we refer the reader to $[\mathbf{1 1}]$. We are here concerned with sufficient conditions on $a(t)$ for the oscillation of $(1)$ when $a(t)$ is allowed to assume negative values for arbitrarily large values of $t$. In the linear case, the well-known Wintner's oscillation theorem states that if $a(t)$ satisfies

$$
\lim _{T \rightarrow \infty} \frac{1}{T} \int_{0}^{T} \int_{0}^{t} a(s) d s d t=+\infty,
$$

then equation (1) is oscillatory for $\gamma=1$, see [8]. Recently, Butler [1] proved that Wintner's theorem remains valid for equation (1) when $\gamma>1$. In the sublinear case, i.e. $0<\gamma<1$, condition (2) can be relaxed to

$$
\limsup _{T \rightarrow \infty} \frac{1}{T} \int_{0}^{T} \int_{0}^{t} a(s) d s d t=+\infty
$$

an earlier result due to Kamenev [4]. We note that condition (3) alone is not sufficient for oscillation even in the linear case, see Willett [9]. However, if we assume in addition to (3) the following condition

$$
\liminf _{t \rightarrow \infty} \int_{0}^{t} a(s) d s=-\lambda>-\infty, \quad \lambda>0,
$$

then we also have oscillation in both the linear and superlinear case. For $\gamma>1$, see [10], and the linear case was settled in Hartman [3].

Several years ago, Kamenev [5] obtained an extension of Wintner's result and proved the following oscillation criterion for the linear equation, i.e. for some $\alpha>1$,

$$
\limsup _{T \rightarrow \infty} \frac{1}{T^{\alpha}} \int_{0}^{T}(T-s)^{\alpha} a(s) d s=+\infty .
$$

Received by the editors September 25, 1985.

1980 Mathematics Subject Classification (1985 Revision). Primary 34C10, 34C15.

Key words and phrases. Oscillation, nonlinear, second order, differential equations. 
It is therefore natural to ask whether condition (5) would imply oscillation also for the more general nonlinear equation (1). The purpose of this note is to prove the following:

THEOREM. Suppose that $a(t)$ satisfies conditions (4) and (5), then equation (1) is oscillatory for all $\gamma>0$.

ProOF. Assume the contrary, then there exists a solution $x(t)$ which may be assumed to be positive on $\left[t_{0}, \infty\right)$ for some $t_{0} \geq 0$. Dividing (1) through by $x^{\gamma}(t)$ and integrating from $t_{0}$ to $t$, we obtain

$$
x^{-\gamma}(t) x^{\prime}(t)+\gamma \int_{t_{0}}^{t}\left(\frac{x^{\prime}(s)}{x^{\beta}(s)}\right)^{2} d s+A(t)=c_{1},
$$

when $\beta=(\gamma+1) / 2, c_{1}=x^{-\gamma}\left(t_{0}\right) x^{\prime}\left(t_{0}\right)$, and $A(t)$ denotes the definite integral $\int_{t_{0}}^{t} a(s) d s$.

We consider two mutually exclusive cases when $x^{\prime} x^{-\beta}(t) \in L^{2}\left(t_{0}, \infty\right)$ and $x^{\prime} x^{-\beta}(t) \notin L^{2}\left(t_{0}, \infty\right)$. In the first case, we note from Schwarz's inequality for $\beta \neq 1$,

$$
\begin{aligned}
\left|x^{1-\beta}(t)-x^{1-\beta}\left(t_{0}\right)\right| & =|1-\beta|\left|\int_{t_{0}}^{t} \frac{x^{\prime}(s)}{x^{\beta}(s)} d s\right| \\
& \leq|1-\beta|\left(t-t_{0}\right)^{1 / 2}\left(\int_{t_{0}}^{t}\left(\frac{x^{\prime}(s)}{x^{\beta}(s)}\right)^{2} d s\right)^{1 / 2} .
\end{aligned}
$$

Since $x^{\prime} x^{-\beta}(t) \in L^{2}\left(t_{0}, \infty\right),(7)$ implies that there exists constants $M$ and $t_{1}>t_{0}$ such that

$$
\left|x^{1-\beta}(t)\right| \leq M t^{1 / 2}, \quad t \geq t_{1} .
$$

Denote $w(t)=x^{\prime}(t) x^{-\gamma}(t)$, then we can rewrite equation (1) as follows:

$$
w^{\prime}(t)+\gamma x^{\gamma-1}(t) w^{2}(t)+a(t)=0, \quad t \geq t_{1} .
$$

Recall that $2(\beta-1)=\gamma-1$, and so using (8) in (9), we obtain

$$
w^{\prime}(t)+M_{0} t^{-1} w^{2}(t)+a(t) \leq 0, \quad t \geq t_{1},
$$

where $M_{0}=\gamma M^{-2}$. Now we multiply (10) through by $(T-t)^{\alpha}$ and integrate by parts to find

$$
\begin{aligned}
\int_{t_{1}}^{T} M_{0} t^{-1}(T-t)^{\alpha} w^{2}(t) d t+ & \alpha \int_{t_{1}}^{T}(T-t)^{\alpha-1} w(t) d t \\
& +\int_{t_{1}}^{T}(T-t)^{\alpha} a(t) d t \leq\left(T-t_{1}\right)^{\alpha} w\left(t_{1}\right) .
\end{aligned}
$$

We can combine the first two terms in (11) by completing the squares under the integral sign and obtain

$$
\begin{aligned}
\int_{t_{1}}^{T} & \left(\sqrt{\frac{M_{0}}{t}}(T-t)^{\alpha / 2} w(t)+\frac{\alpha}{2} \sqrt{\frac{t}{M}}(T-t)^{\alpha / 2-1}\right)^{2} d t \\
& -\frac{\alpha^{2}}{4 M_{0}} \int_{t_{1}}^{T} t(T-t)^{\alpha-2} d t+\int_{t_{1}}^{T}(T-t)^{\alpha} a(t) d t \leq\left(T-t_{1}\right)^{\alpha} w\left(t_{1}\right) .
\end{aligned}
$$


We note that the first integral in (12) is nonnegative and observe that, for $\alpha>1$,

$$
\lim _{T \rightarrow \infty} \frac{1}{T^{\alpha}} \int_{t_{1}}^{T} t(T-t)^{\alpha-2} d t=\frac{1}{\alpha(\alpha-1)},
$$

so the second integral in (12) tends to a finite limit. Hence condition (5) would produce the desired contradiction in (12).

Next we consider $x^{\prime} x^{-\beta}(t) \notin L^{2}\left(t_{0}, \infty\right)$. Using condition (4) in (6), we deduce that $x^{\prime}(t)<0$ for sufficiently large $t$, say $t \geq t_{1} \geq t_{0}$. We now estimate (6) as follows:

$$
-\frac{x^{\prime}(t)}{x^{\gamma}(t)} \geq-\left(c_{1}+\lambda\right)+\gamma \int_{t_{1}}^{t} \frac{x^{2}(s)}{x^{\gamma+1}(s)} d s .
$$

By assumption, we can choose $t_{2} \geq t_{1}$ such that

$$
\gamma \int_{t_{1}}^{t_{2}} \frac{x^{\prime 2}(s)}{x^{\gamma+1}(s)} d s=1+c_{1}+\lambda
$$

For $t \geq t_{2}$, we multiply (13) through by the following positive term

$$
-\gamma \frac{x^{\prime}(t)}{x(t)}\left\{-\left(c_{1}+\lambda\right)+\gamma \int_{t_{1}}^{t} \frac{x^{\prime 2}(s)}{x^{\gamma+1}(s)} d s\right\}^{-1}
$$

and integrate from $t_{2}$ to $t$ to obtain

$$
\log \left(-\left(c_{1}+\lambda\right)+\gamma \int_{t_{1}}^{t} \frac{x^{\prime 2}(s)}{x^{\gamma+1}(s)} d s\right) \geq \gamma \int_{t_{2}}^{t} \frac{-x^{\prime}(s)}{x(s)} d s=\gamma \log \frac{x\left(t_{2}\right)}{x(t)}
$$

which together with (13) yields

$$
-x^{\prime}(t) / x^{\gamma}(t) \geq x^{\gamma}\left(t_{2}\right) / x^{\gamma}(t)
$$

from which it follows that $x^{\prime}(t) \leq-x^{\gamma}\left(t_{2}\right)<0$, contradicting the assumption that $x(t)>0$. This completes the proof.

REMARK 1. Condition (4) has also been used by Onose [7] in connection with other oscillation theorems for the sublinear equation.

REMARK 2. The same technique can be used to extend oscillation criterion for an equation with damped term, see recent results of Yan [12] and Yeh [14].

REMARK 3. Kamenev's condition (5) is useful only when $a(t)$ becomes negative for largest values of $t$. If $a(t)$ is eventually nonnegative, then (5) is equivalent to the divergence of $A(t)$. Such results as $[2,13]$ are therefore not very meaningful.

REMARK 4. More recently, extensive generalizations of oscillation results for the linear equation to the nonlinear equation (1) were also given in [6].

\section{REFERENCES}

1. G. J. Butler, Integral averages and the oscillation of second order ordinary differential equations, SIAM J. Math. Anal. 11 (1980), 190-200.

2. S. R. Grace and B. S. Lalli, Án oscillation criterion for nth order nonlinear differential equations with functional arguments, Canad. Math. Bull. 26 (1983), 35-40.

3. P. Hartman, On nonoscillatory linear differential equations of second order, Amer. J. Math. 74 (1952), 389-400.

4. I. V. Kamenev, Certain specifically nonlinear oscillation theorems, Mat. Zametki 10 (1971), 129-134. (Russian) 
5. _ Integral criterion for oscillations of linear differential equations of second order, Mat. Zametki 23 (1978), 249-251.

6. M. K. Kwong and J. S. W. Wong, Linearization of second order nonlinear oscillation theorems, Trans. Amer. Math. Soc. 279 (1983), 705-772.

7. H. Onose, Oscillation criteria for second order nonlinear differential equations, Proc. Amer. Math. Soc. 51 (1975), 67-73.

8. A. Wintner, A criterion of oscillatory stability, Quart. Appl. Math. 7 (1949), 114-117.

9. D. Willett, Classification of second order linear differential equations with respect to oscillation, Adv. in Math. 3 (1969), 594-623.

10. J. S. W. Wong, A second order nonlinear oscillation theorem, Proc. Amer. Math. Soc. 40 (1973), 487-491.

11. __, On the generalized Emden-Fowler equation, SIAM Rev. 17 (1975), 339-360.

12. J. Yan, $A$ note on an oscillation criterion for an equation with damped term, Proc. Amer. Math. Soc. 90 (1984), 277-280.

13. C. C. Yeh, An oscillation criterion for second order nonlinear differential equations with functional arguments, J. Math. Anal. Appl. 76 (1980), 72-76.

14. __ Oscillation theorems for nonlinear second order differential equations with damped terms, Proc. Amer. Math. Soc. 84 (1982), 397-402.

China DYeing Works, LTD., 819 SWIRE HOUSE, HONG KONG KONG

Department of Mathematics, University of Hong Kong, Pokfulman, Hong 